\title{
A prospective study of hip revision surgery using the Exeter long-stem prosthesis: function, subsidence, and complications for 57 patients
}

\author{
K. Randhawa $\cdot$ F. S. Hossain $\cdot$ B. Smith . \\ Cyril Mauffrey $\cdot$ T. Lawrence
}

Received: 26 February 2009/Accepted: 28 September 2009/Published online: 24 October 2009

(C) Springer-Verlag 2009

\begin{abstract}
Background The long-stem Exeter femoral component is commonly used in revision hip surgery. Subsidence of the femoral stem in primary hip arthroplasty has been studied extensively, but much less is known about its significance in revision surgery. This prospective study examined the relationship between radiological subsidence, Western Ontario and McMaster (WOMAC) osteoarthritis index pain score, patient satisfaction and complication rates for the long-stem Exeter hip prosthesis.

Materials and methods Data was prospectively collected for a single-surgeon series of 96 patients undergoing revision surgery with a mean follow-up period of 36 months. Pre- and post-operative clinical evaluation was carried out using the validated WOMAC osteoarthritis index. Radiographic evaluation was carried out on magnificationadjusted digital radiographic images.

Results Data from 57 patients were analysed. The mean rate of subsidence recorded was $0.43 \mathrm{~mm} / \mathrm{year}$, with a mean total subsidence of $0.79 \mathrm{~mm}$ [95\% confidence interval (CI) $0.57-1.01]$ at 36.3 months. There was no correlation between subsidence and post-operative WOMAC score, complication rate or patient satisfaction. There was a statistically significant reduction between pre-operative and post-operative WOMAC scores, with means of
\end{abstract}

K. Randhawa · F. S. Hossain - B. Smith · C. Mauffrey ·

T. Lawrence

The Heart of England NHS Trust, Lode Lane Solihull,

Birmingham, UK

C. Mauffrey $(\bowtie)$

Birmingham Heartlands and Solihull Hospital, Lode Lane, 456 Warwick Road, Solihull, West Midlands B911AG, UK

e-mail: cmauffrey@yahoo.com
33.5 and 10.7 , respectively $(P<0.001)$, and high patient satisfaction.

Conclusion Our subsidence rates for long-stem revision femoral components are lower than the published data but demonstrate the same plateau. Radiographic subsidence does not appear to relate to functional outcome or complication rates in our data.

Keywords Exeter long stem - Revision hip arthroplasty · Subsidence

\section{Introduction}

With an increasing number of younger patients undergoing total hip arthroplasty and the finite lifespan of prosthesis, the incidence of revision hip surgery is on the rise. A previous study by Coyte et al. projected a $5.1 \%$ annual growth in the number of revision hip arthroplasties [1]. Revision arthroplasty is associated with higher complications rates [2] and a smaller improvement in functional outcome. Common causes for revision surgery include prosthesis loosening (both septic and aseptic), recurrent dislocation, femoral and acetabular osteolysis and periprosthetic fracture of the femur. The method of hip reconstruction is usually individualised to each patient depending on the extent of bone loss, surgeon experience and anticipated patient lifespan. The revision femoral stem is usually longer than the retrieved stem in order to bypass the deficient proximal defect and achieve a tighter diaphyseal fixation. Revision for an infected prosthesis is usually performed in two stages. In aseptic loosening, cementwithin-cement is sometimes employed where removal of the cement mantle proves difficult, thus avoiding the complications of removing acrylic cement (i.e. femoral 
fracture, reduced bone stock and blood loss). This is still an area of much debate [3, 4].

Many options are now available for complex femoral reconstruction, including the use of long stems, uncemented or cemented implants, hydroxyapatite (HA)-coated implants, impaction grafting, massive allografts or proximal femoral replacement type implants. These prosthetic implants in a variety of stem lengths demonstrate varying clinical and radiological success [5-7]. Long-stem femoral components have been shown to be favourable in revision hip arthroplasty [8, 9]. The Exeter stem, however, enjoys a significantly long survivorship [9] and is a popular choice amongst orthopaedic surgeons in both primary and revision total hip arthroplasty. We report a cohort of 57 patients with a mean follow-up of 36 months following revision total hip arthroplasty using cemented Exeter long-stem implant. This paper focuses on functional outcomes using the Western Ontario and McMaster (WOMAC) osteoarthritis index pain score, complication rates and radiological subsidence.

\section{Materials and method}

A prospective cohort study was undertaken of a singlesurgeon series at our institution from 1999 to 2007. Revision cemented hip arthroplasty was performed by the senior author (TL). Ninety-six, Exeter double-tapered long-stem femoral prostheses (Stryker/Howmedia Osteonics Newbury, UK) were inserted into 96 patients. Informed consent was obtained prior to patient inclusion in our study. The study was planned and implemented in accordance with the ethical standards of the Helsinki Declaration as amended in 2000 and was approved by our institution's ethics committee. Our indications for long-stem femoral revision were as follows: stem loosening with distal osteolysis, intraoperative femoral perforation, intra-operative femoral window, periprosthetic fractures, proximal segmental bone-stock deficiencies, lack of proximal femoral cancellous bone and complex reconstruction with re-alignment osteotomy.

\section{Patient demographics}

Mean patient age was 73 (range 37-94) years at the time of surgery. There were $42(43.8 \%)$ men and $54(56.2 \%)$ women. The indications for revision were femoral and acetabular loosening in 27 patients (28.1\%), femoral loosening only in $28(29.1 \%)$, acetabular loosening only in six $(6.25 \%)$, infection in $13(13.5 \%)$, acetabular dislocation in two $(2.0 \%)$, femoral osteolysis in four $(4.16 \%)$, global osteolysis in seven $(7.2 \%)$, periprosthetic fracture in eight $(8.3 \%)$ and mixed causes in one $(1 \%)$.
Surgical technique

All operations were performed at the Heart of England NHS Trust by the senior author in dedicated orthopaedic theatres. A modified Hardinge approach was used in all patients. The affected components were removed along with the cement, debris and fibrous material. Of the extracted femoral prosthesis, $58(57.4 \%)$ were cemented and 38 were uncemented. The femoral canal was curetted and reamed, and a range of broach sizes was tried. Impaction grafting was used in cases of cancellous bone stock deficiency. Sclerotic bone was removed and replaced with impaction grafting. Distal plugs were used to fill femoral windows. Third-generation cementing technique using pulsed lavage, tamponade and vacuum mixing with pressurization of gentamicin-loaded cement [SIMPLEX (Stryker, Newbury, UK)] was standard for all patients. A total of six cement mixes were routinely used when cementing the femoral component. One patient received PALACOS cement (Zimmer, UK). Two types of acetabular components were used in 96 patients who underwent total revision arthroplasty. Eighty-eight (93.6\%) patients received the Elite cup (Depuy, Leeds, UK), and eight (6.4\%) patients received the Ogee cup (Depuy). Eighty-eight $(91.6 \%)$ patients received a $28-\mathrm{mm}$ femoral head, four $(4 \%)$ received a $32-\mathrm{mm}$ head and four $(4 \%)$ received a $22.2-\mathrm{mm}$ head. All patients received prophylactic parenteral administration of the antibiotics flucloxacillin $1 \mathrm{~g}$ and gentamicin $160 \mathrm{mg}$ pre-operatively, as well as 10 days of post-operative parenteral administration of flucloxacillin $500 \mathrm{mg}$ QDS. Antithrombotic prophylaxis consisted of tedgradcoman (T.E.D.S) stockings and subcutaneous administration of enoxaparin (Clexane, SanofiAventis) injection daily for 5 days, followed by aspirin $75 \mathrm{mg}$ daily for 6 weeks. Post-operative rehabilitation consisted of 6 weeks of non-weight bearing for peri-prosthetic fractures, followed by partial/full weight bearing or 1 week non-weight bearing followed by full weight bearing for the other indications.

\section{Clinical evaluation}

Pre-operatively, patients underwent clinical evaluation and were assessed according to the validated WOMAC osteoarthritis score [10]. Endpoints for the study were re-revision, pain, stiffness, function and complications. Data was collected prospectively at 6 and 12 months post-operatively and then yearly. Interview and clinical examination were carried out in the outpatient clinic. The WOMAC osteoarthritis index and a patient satisfaction questionnaire were used at each follow-up appointment. Patients were asked if they would recommend the procedure to a friend or relative in a four-point scale (definitely not, probably not, possibly yes, definitely yes). 


\section{Radiological evaluation}

All patients were followed up with pre- and post-operative radiographic investigations. All investigations were performed at our hospital and included standardised anteriorposterior and lateral projections of the hip. Two observers evaluated the radiographs. Subsidence was evaluated by comparing the immediate post-operative radiographic images with those obtained at follow-up. All radiographs were digitalised. Subsidence was measured as a vertical drop of the stem, characterised by a radiolucent line at the proximal most aspect of the cement-prosthesis interface. Magnification was adjusted based on the known diameter of the prosthetic femoral head. Our measurements were aided by the use of an on-screen calliper and protractor software (www.iconico.com).

\section{Statistics}

Statistical analysis was carried out using the XLSTAT module (Addinsoft, NY, USA) for Microsoft Excel. The paired Students' $t$ test and analysis of variance (ANOVA) for parametric data, set at a $0.05 \%$ significance level, was used for data analysis. Linear regression analysis was used to correlate Paproski scores to subsidence and percentage WOMAC score improvements to subsidence.

\section{Results}

\section{Follow-up}

At review, 13 patients had died. The cause of death on review of patient's notes was not directly related to the revision. Eleven patients were lost to follow-up, and four patients did not have adequate follow-up time (less than 6 months), leaving 68 patients for analysis with a mean follow-up time of 36 (range 6-84) months (Fig. 1).

\section{Complications}

There were four cases $(4.1 \%)$ of superficial infection, which were treated by intra-operative washout. Three patients $(3.1 \%)$ suffered deep infection, two of whom underwent two-stage re-revision surgery (at 34 and 33 months post-operatively), and the other patient underwent a washout with prosthesis retention. One patient presented with loosening of both components and underwent a re-revision 5 years after the primary operation. Four patients $(4.1 \%)$ presented with peri-prosthetic fractures; of these, three underwent re-revision femoral stem surgery, and one was treated conservatively. Two patients presented with deep vein thrombosis and were treated with

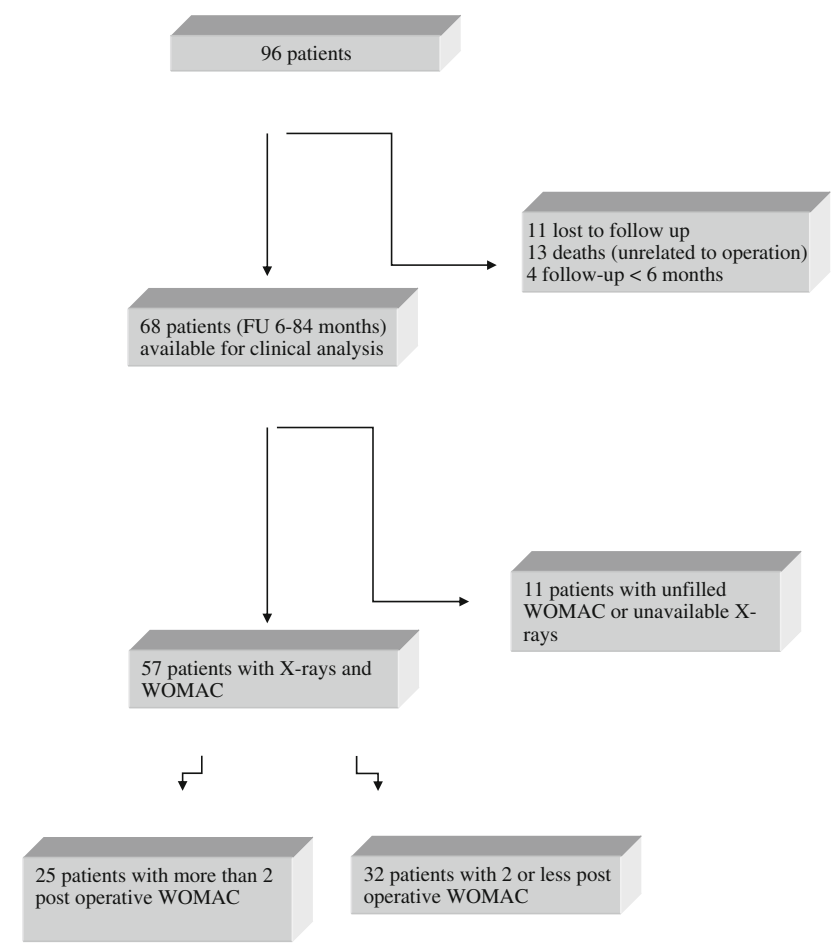

Fig. 1 Patients recruited to the study

anti-coagulation therapy. One patient suffered recurrent dislocations and underwent an acetabular revision (Table 1).

Clinical findings

From the 68 patients analysed, only 57 filled in the WOMAC questionnaire, with an average follow-up of 36.3 (range 6-84) months (Fig. 1). Pre-operatively, the average WOMAC score was 33.5 [95\% confidence interval (CI) 31.0-36.0]. At the time of the latest follow-up, the average WOMAC score was 10.7 (CI 8.4-13.0), registering an improvement of 22.8. This was found to be statistically significant $(P<0.001$, CI 18.93-26.12) (Table 2).

Of these 57 patients, 25 had a recorded WOMAC score at more than two occasions post-operatively (12, 24, 36 and/or 48 months post-operatively). We analysed this

Table 1 Complications associated with our cohort of patients

\begin{tabular}{ll}
\hline Type of complication & $\begin{array}{l}\text { Number } \\
\text { (percentage of all patients) }\end{array}$ \\
\hline Superficial infection & $4(4.1)$ \\
Deep infection & $3(3.1)$ \\
Loosening (both components) & $1(1)$ \\
Peri-prosthetic fracture & $4(4.1)$ \\
Deep vein thrombosis & $2(2.1)$ \\
Recurrent dislocation & $1(1)$ \\
\hline
\end{tabular}


Table 2 Western Ontario and McMaster (WOMAC) osteoarthritis index pain outcome scores, subsidence and follow-up times

\begin{tabular}{llc}
\hline Result & Mean & $\begin{array}{l}\text { 95\% confidence } \\
\text { interval }\end{array}$ \\
\hline Average follow-up & $\begin{array}{c}36.3 \text { months } \\
\text { (range 6-84) }\end{array}$ & - \\
Pre-operative WOMAC & 33.5 & $31.0-36.0$ \\
Post-operative WOMAC & 10.7 & $8.4-13.0$ \\
Subsidence & $0.79 \mathrm{~mm}$ & $0.57-1.01$ \\
& $\quad$ (range 0-2.81) & \\
Rate of subsidence & $0.43 \mathrm{~mm} /$ year & $0.24-0.62$ \\
\hline
\end{tabular}

${ }^{a}$ Results for 40 patients, as 17 patients showed no subsidence

subgroup to ascertain whether there was a trend of postoperative WOMAC score with time. There was a trend to improvement in terms of overall WOMAC scores (Fig. 2). In considering the WOMAC pain scores, graphical analysis suggested some worsening in pain at late follow-up (Fig. 3a). However, following statistical analysis using ANOVA, this trend was found to be non-significant $(P=0.777)$. The same analysis was performed on WOMAC activity scores (Fig. 3b). There was no significant difference in WOMAC activity scores over time $(P=0.751)$. Fifty-seven patients answered the WOMAC satisfaction questionnaire, of whom only one patient would not recommend revision arthroplasty to a friend. This patient had to undergo a re-revision total hip arthroplasty due to resistant infection. Sixty patients answered "definitely yes", six patients answered "probably yes", and one patient answered "probably not" to the same question.

\section{Radiological findings}

Of the 57 patients available for X-ray analysis and complete WOMAC questionnaires, 17 cases showed no subsidence. The mean subsidence for the 57 patients was $0.79 \mathrm{~mm}$ (CI 0.57-1.01, range 0-2.81). The mean rate of subsidence was $0.43 \mathrm{~mm} /$ year (CI 0.24-0.62), as outlined in Table 2 .

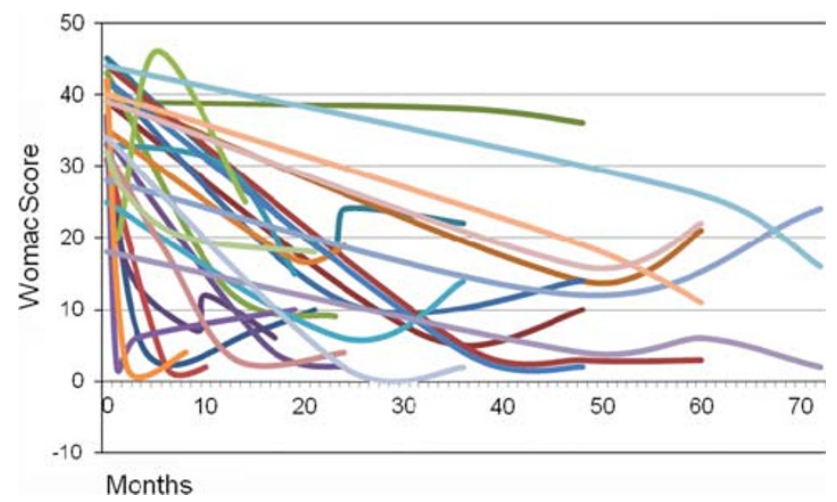

Fig. 2 Western Ontario and McMaster (WOMAC) score over time
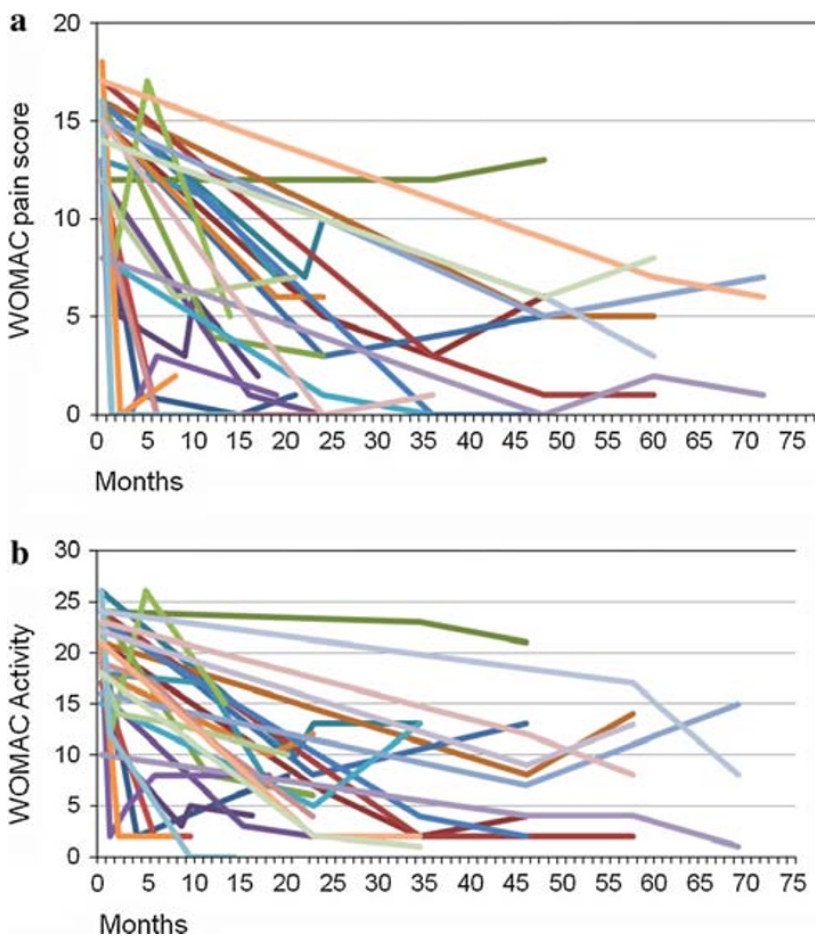

Fig. 3 Western Ontario and McMaster (WOMAC) pain score (a) and activity score (b) over time

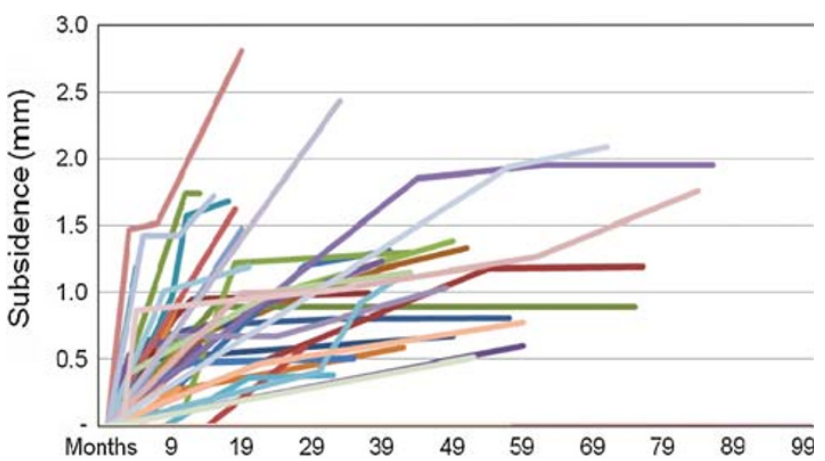

Fig. 4 Subsidence over time for all patients

The patient with the largest subsidence $(2.81 \mathrm{~mm})$ had a postoperative WOMAC score of 6, down from 37 at 21 months post-operatively, and was highly satisfied with the procedure. The subsidence-time graph for all the patients is shown in Fig. 4. Of the 57 patients with subsidence data, 23 showed a subsidence of between 1.0 and $2.81 \mathrm{~mm}$ at an average of 39.2 months' follow-up. Only one of these patients, who recorded a subsidence of $2.09 \mathrm{~mm}$, suffered from a complication (superficial wound infection).

We performed a regression analysis to assess the correlation between the percentage change in WOMAC score and subsidence and found no correlation between these two variables (Fig. 5: coefficient of determination, $R^{2}=0.0015$ ). In addition, we performed a regression 


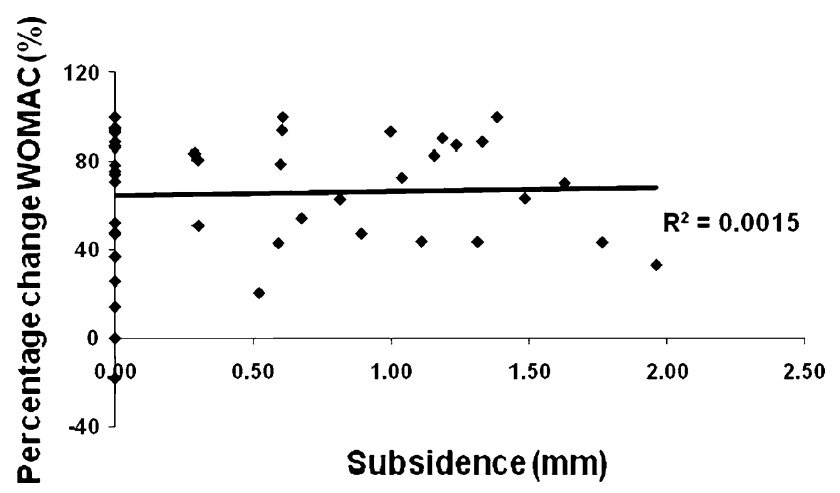

Fig. 5 Percentage change in Western Ontario and McMaster (WOMAC) scores versus subsidence

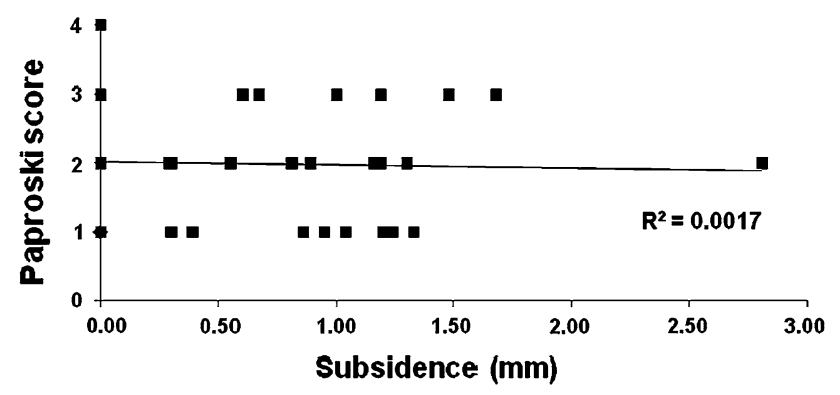

Fig. 6 Paproski scores versus subsidence

analysis by plotting Paproski femoral scores against subsidence and found no correlation between the two variables. (Fig. $6, R^{2}=0.0017$ ).

\section{Discussion}

Revision hip arthroplasty is a technically difficult procedure. As younger patients undergo primary hip arthroplasty, the age of the revision hip patient is falling. Needless to say, failure rates in revision hip arthroplasty are higher than in primary hip surgery [11-13]. Surgeons are faced with the task of augmenting the reduced bone stock and weakened soft tissues. The options for revision are vast. Augmenting femoral bone loss is the main difficulty in achieving stable fixation. Cemented revision with impacted cancellous allograft is one of the ways to overcome this difficulty [14-16]. Previous studies on cemented long-stem revisions have shown high failure rates $[15,17$, 18]; however, this can be attributed to older cementing techniques. Cementless revision techniques are suitable in some cases. However, results are unfavourable [19, 20], and patients often complain of thigh pain and must undergo long periods of post-operative immobility [21].

In our study, all patients received the Exeter long stem. At 220-mm long, it has the advantage of bridging the bony deficiencies in the femoral canal. Seven patients underwent re-revision surgery; three for peri-prosthetic fractures, two for deep infection, one for aseptic loosening and one for recurrent dislocation. Periprosthetic fractures are a common complication of revision hip surgery. In our series, this was secondary to falls. Only one patient suffered aseptic loosening. This occurred 5 years after the initial surgery. In comparison, the aseptic loosening rate in other studies varied between $7 \%$ and $26 \%$ [8-10, $15,16,20]$.

Subsidence is defined as the inferior migration of the femoral stem following total hip arthroplasty. It is a phenomenon that applies to all stems, regardless of surface finish, presence of cement and type of stem and is due to axial forces acting on the prosthesis. Subsidence for primary total hip arthroplasty has been studied extensively. Less is known about its significance in revision hip arthroplasty. Subsidence of a femoral stem is governed by a number of factors. It was initially thought that an unpolished, roughened surface would provide stability and prevent femoral-stem loosening. These stems enjoyed good to excellent results and demonstrated minimal subsidence [22]. On the other hand, polished tapered stems make good use of cement creep and stress relaxation of cement to achieve stability [23]. The most common pattern is rapid initial subsidence followed by secondary stabilisation [24, 25]. This characteristic, however, is stem dependent [30]. Excessive subsidence has been shown to predict early failure $[26,27]$. Cement viscosity was found not to significantly affect subsidence [28], and neither was the crosssectional shape of prosthesis [29]. Subsidence in revision femoral stems is further affected by impaction grafting and the integrity of the cortical envelope [30].

Our subsidence rate averaged $0.79 \mathrm{~mm}$ with a mean follow-up of 36.3 months. Seventeen hips exhibited no subsidence, with a mean follow-up of 34.6 months. Overall, these values for subsidence are far lower compared with published results. Eldridge et al. reported nine cases of massive early subsidence following cemented revision total hip arthroplasty [31]. Meding et al. found a mean subsidence of $10.1 \mathrm{~mm}$ in 13 of their 28 patients [32]. Other studies have reported similar results [33,34]. Our good results might be attributed to surgical cementing technique. Furthermore, our subsidence figures are comparable with those of primary hip arthroplasty.

There was no correlation between subsidence and WOMAC scores. This is likely due to our low subsidence figures. Surprisingly, bone loss (as graded by Paproski femoral score) did not affect subsidence. The patient who had the least bone stock prior to surgery did not have any subsidence 60 months post-operatively. We attribute this result to good cementing and allograft impaction techniques. 
The gold standard for measuring subsidence is by Roentgen stereo-photogrammetric analysis (RSA) [35]. This represents an accurate method of determining threedimensional stem migration and has been used in numerous studies. However, it is time consuming, expensive and requires pre-operative implantation of tantalum beads. Our method may not be as accurate as RSA but allows the surgeon to measure subsidence rapidly and to reproduce the measurements with future X-rays. This will allow the surgeon to differentiate the high-risk group of patients who may require more frequent follow-up from those who have stable hips and may be discharged from follow-up. Further studies are required to evaluate this theory. We confirm that small subsidence $(<3.0 \mathrm{~mm})$ is not significant. Our method allows accurate measurement of subsidence that could be clinically significant.

The WOMAC score is a patient-oriented functional questionnaire. It takes into account patient perception of pain and activity levels both pre- and post-surgery. Our results show that after an initial improvement of WOMAC scores post-operatively, some patients show a slight worsening of their WOMAC scores. ANOVA testing revealed no significant difference between the post-operative scores at 12, 24, 36 and 48 months. A similar trend was seen for WOMAC activity score. Once again, there was no significant difference between the score at the various post-operative periods. Prior studies have shown little correlation between subsidence and clinical scoring provided there was no loosening [36]. In our study, patients satisfaction score was excellent. All but one patient would recommend the procedure to a friend.

In conclusion, the Exeter long femoral stem is an excellent option in revision hip arthroplasty. Our subsidence rates for long-stem revision femoral components are lower than the published data but demonstrate the same plateau. Radiographic subsidence does not appear to relate to functional outcome or complication rates in our data, but other studies show subsidence to be a significant indicator of premature prosthesis failure. Longer follow-up will be required to see if this is the case in our prospective cohort. Our method of measuring subsidence is simple, reproducible and can be used in the clinical setting.

Conflict of interest statement We certify that there is no actual or potential conflict of interest in relation to this article.

\section{References}

1. Coyte PC, Young W, Williams JI (1998) Devolution of hip and knee replacement surgery? Can J Plast Surg 39(5):373-378

2. Hungerford DS, Jones LC (1988) The rationale of cementless revision of cemented arthroplasty failures. Clin Orthop Relat Res 235:12-24
3. Li P, Ingle P, Dowell J (1996) Cement within cement revision hip arthroplasty; should it be done? J Bone Joint Surg Br 78(5): 809-811

4. Mandziak DG, Howie DW et al (2007) Cement-within-cement stem exchange using the collarless polished double-taper stem. J Arthroplasty 22(7):1000-1006

5. D'Lima DD, Oishi CS et al (1998) 100 cemented versus 100 noncemented stems with comparison of 25 matched pairs. Clin Orthop Relat Res 348:140-148

6. Tsiridis E, Narvani AA, Haddad FS et al (2004) Impaction femoral allografting and cemented revision for periprosthetic femoral fractures. J Bone Joint Surg Br 86(8):1124-1132

7. Garbuz DS, Toms A et al (2006) Improved outcome in femoral revision arthroplasty with tapered fluted modular titanium stems. Clin Orthop Relat Res 453:199-202

8. Hultmark P, Karrholm J et al (2000) Cemented first-time revisions of the femoral component: prospective 7 to 13 years' follow-up using second-generation and third-generation technique. J Arthroplasty 15(5):551-561

9. Franklin J, Robertsson O et al (2003) Revision and complication rates in 654 Exeter total hip replacements, with a maximum follow-up of 20 years. BMC Musculoskelet Disord 4:6

10. Bellamy N (2005) The WOMAC knee and hip osteoarthritis indices: development, validation, globalization and influence on the development of the AUSCAN hand osteoarthritis indices. Clin Exp Rheumatol 23(5 Suppl 39):S148-S153

11. Callaghan JJ, Salvati EA, Pellicci PM et al (1985) Two to five year results of revision total hip replacement. AAOS 52nd Annual Meeting

12. Pellicci PM, Wilson PD Jr et al (1982) Revision total hip arthroplasty. Clin Orthop Relat Res 170:34-41

13. Pellicci PM, Wilson PD Jr et al (1985) Long-term results of revision total hip replacement. A follow-up report. J Bone Joint Surg Am 67(4):513-516

14. Gie GA, Linder L et al (1993) Impacted cancellous allografts and cement for revision total hip arthroplasty. J Bone Joint Surg Br 75(1):14-21

15. Ling RS, Timperley AJ, Linder L (1993) Histology of cancellous impaction grafting in the femur. A case report. J Bone Joint Surg Br 75(5):693-696

16. Slooff TJ, Buma P et al (1996) Acetabular and femoral reconstruction with impacted graft and cement. Clin Orthop Relat Res 324:108-115

17. Kavanagh BF, Fitzgerald RH Jr (1987) Multiple revisions for failed total hip arthroplasty not associated with infection. J Bone Joint Surg Am 69(8):1144-1149

18. Mulroy WF, Harris WH (1996) Revision total hip arthroplasty with use of so-called second-generation cementing techniques for aseptic loosening of the femoral component. A fifteen-yearaverage follow-up study. J Bone Joint Surg Am 78(3):325-330

19. Berry DJ, Harmsen WS et al (1995) Survivorship of uncemented proximally porous-coated femoral components. Clin Orthop Relat Res 319:168-177

20. Mulliken BD, Rorabeck CH et al (1996) Uncemented revision total hip arthroplasty: a 4-to-6-year review. Clin Orthop Relat Res 325:156-162

21. Callaghan JJ, Dysart SH et al (1988) The uncemented porouscoated anatomic total hip prosthesis. Two-year results of a prospective consecutive series. J Bone Joint Surg Am 70(3):337-346

22. Espehaug B, Havelin LI et al (1995) Early revision among 12, 179 hip prostheses. A comparison of 10 different brands reported to the Norwegian Arthroplasty Register, 1987-1993. Acta Orthop Scand 66(6):487-493

23. Fowler JL, Gie GA et al (1988) Experience with the Exeter total hip replacement since 1970. Orthop Clin North Am 19(3): 477-489 
24. Karrholm J, Hultmark P et al (1999) Subsidence of a non-polished stem in revisions of the hip using impaction allograft. Evaluation with radiostereometry and dual-energy X-ray absorptiometry. J Bone Joint Surg Br 81(1):135-142

25. Alfaro-Adrian J, Gill HS et al (1999) Cement migration after THR. A comparison of Charnley elite and Exeter femoral stems using RSA. J Bone Joint Surg Br 81(1):130-134

26. Walker PS, Mai SF et al (1995) Prediction of clinical outcome of THR from migration measurements on standard radiographs. A study of cemented Charnley and Stanmore femoral stems. J Bone Joint Surg Br 77(5):705-714

27. Krismer M, Biedermann R et al (1999) The prediction of failure of the stem in THR by measurement of early migration using EBRA-FCA. Einzel-Bild-Roentgen-Analyse-femoral component analysis. J Bone Joint Surg Br 81(2):273-280

28. Nelissen RG, Garling EH et al (2005) Influence of cement viscosity and cement mantle thickness on migration of the Exeter total hip prosthesis. J Arthroplasty 20(4):521-528

29. Ek ET, Choong PF (2005) Comparison between triple-tapered and double-tapered cemented femoral stems in total hip arthroplasty: a prospective study comparing the C-Stem versus the Exeter universal early results after 5 years of clinical experience. J Arthroplasty 20(1):94-100
30. Knight JL, Helming C (2000) Collarless polished tapered impaction grafting of the femur during revision total hip arthroplasty: pitfalls of the surgical technique and follow-up in 31 cases. J Arthroplasty 15(2):159-165

31. Eldridge JD, Smith EJ et al (1997) Massive early subsidence following femoral impaction grafting. J Arthroplasty 12(5): $535-540$

32. Meding JB, Et MA et al (1997) Impaction bone-grafting before insertion of a femoral stem with cement in revision total hip arthroplasty. A minimum two-year follow-up study. J Bone Joint Surg Am 79(12):1834-1841

33. Masterson EL, Masri BA et al (1997) The cement mantle in the Exeter impaction allografting technique. A cause for concern. J Arthroplasty 12(7):759-764

34. Elting JJ, Mikhail WE et al (1995) Preliminary report of impaction grafting for exchange femoral arthroplasty. Clin Orthop Relat Res 319:159-167

35. Selvik G (1989) Roentgen stereophotogrammetry. A method for the study of the kinematics of the skeletal system. Acta Orthop Scand Suppl 232:1-51

36. Schreurs BW, Arts JJ et al (2005) Femoral component revision with use of impaction bone-grafting and a cemented polished stem. J Bone Joint Surg Am 87(11):2499-2507 